\title{
THE EXACT CARDINALITY OF THE SET OF INVARIANT MEANS ON A GROUP
}

\author{
CHING $\mathrm{CHOU}^{1}$
}

\begin{abstract}
The purpose of this note is to show that if $G$ is an infinite amenable group then $G$ has exactly $2^{2^{|G|}}$ invariant means where $|G|$ denotes the cardinality of $G$.
\end{abstract}

Let $G$ be a discrete group, $m(G)$ the space of bounded real functions on $G$ with the sup norm. For $x \in G$ and $f \in m(G), l_{x} f$ and $r_{x} f$ are defined by $\left(l_{x} f\right)(y)=f(x y)$ and $\left(r_{x} f\right)(y)=f(y x)$, respectively, $y \in G . \mu \in m(G)^{*}$ is called a left (right) invariant mean on $m(G)$ if $\mu \geqslant 0,\|\mu\|=1$ and $\mu\left(l_{x} f\right)$ $=\mu(f)\left(\mu\left(r_{x} f\right)=\mu(f)\right)$ for $x \in G$ and $f \in m(G)$. We shall denote the set of left (right) invariant means on $G$ by $M L(G)(M R(G))$. Let $M(G)$ $=M L(G) \cap M R(G)$, the set of two-sided invariant means, and $M^{\sim}(G)$ $=\{\mu \in M(G): \mu$ is inversion invariant $\} .\left(\mu \in m(G)^{*}\right.$ is said to be inversion invariant if $\mu(f)=\mu\left(f^{\prime}\right)$ for each $f \in m(G)$ where $f$ is defined by $f^{\sim}(x)=f\left(x^{-1}\right)$.) $G$ is said to be amenable if there is at least one left invariant mean on $m(G)$. When $G$ is amenable, $M^{\sim}(G) \neq \varnothing$, cf. [3].

If $X$ is a set, $|X|$ will denote the cardinality of $X$. If $G$ is a finite group then $m(G)$ has a unique left invariant mean $\mu: \mu(f)=(1 /|G|) \sum\{f(x): x \in G\}$. E. Granirer [5] proved that this is the only case that $M L(G)$ is a singleton. He actually proved that if $G$ is infinite amenable then the vector space spanned by $M L(G)$ is infinite dimensional. In [1] we showed that if $G$ is infinite amenable then $|M L(G)| \geqslant 2^{c}$ where $c$ is the cardinality of the continuum. It implies that the linear span of $M L(G)$ is not even norm separable.

On the other hand, we are unable to find a theorem in the literature stating that $M(G)$ is not a singleton if $G$ is infinite. But if $G$ is assumed to be countably infinite and amenable then we do know that $|M(G)| \geqslant 2^{c}[2$, Theorem 4.3].

Note that if $G$ is infinite then $\left|m(G)^{*}\right|=2^{2^{|G|}}$. Since $M L(G) \subset m(G)^{*}$, $|M L(G)| \leqslant 2^{2^{|G|}}$. Our main result is

THEOREM 1. Let $G$ be an infinite amenable group. Then $|M L(G)|=|M(G)|$ $=\left|M^{\sim}(G)\right|=2^{2^{|G|}}$.

Clearly it is enough to show that $\left|M^{\sim}(G)\right| \geqslant 2^{2^{|G|}}$. In [2, Theorem 4.3] it is

Received by the editors July 11, 1974 and, in revised form, January 9, 1975.

AMS (MOS) subject classifications (1970). Primary 43A07.

Key words and phrases. Invariant means, amenable groups, cardinalities.

${ }^{1}$ Supported in part by NSF grant P034190-001. 
proved that if $G$ is countably infinite then there is a one-one mapping of a set T⿱ $㇒$ of cardinality $2^{c}=2^{2^{n_{0}}}$ into $M(G)$. The fact that the sequence $\left\{U_{n}\right\}$ in [2] can be chosen to be symmetric implies that the image of $\mathbb{R}$ is actually contained in $M^{\sim}(G)$; see [2, pp. 450-451]. Therefore it remains to consider the case $|G|>\aleph_{0}$. We shall actually prove the stronger

THEOREM 2. Let $G$ be an uncountable amenable group. Then there is a family of subsets $\left\{E_{\theta}\right\}_{\theta \in \Theta}$ of $G,|\Theta|=2^{|G|}$, such that each set function on $\left\{E_{\theta}\right\}_{\theta \in \Theta}$ with values in $[0,1]$ is the restriction of an element of $M^{\sim}(G)$.

Since there are $2^{2^{|G|}}$ such set functions, the uncountable case of Theorem 1 follows directly from the above theorem. To prove Theorem 2 we shall follow closely the steps of the proof of the well-known theorem of Kakutani and Oxtoby [7]. Their theorem is also reproduced in full in Hewitt and Ross $[6, \S 16]$. Therefore we shall skip some of the details. Our notation is similar to that of [6].

From now on $G$ will denote a fixed amenable group with $|G|>\kappa_{0}$. Let $T$ be the set of mappings $x \rightarrow a x, x \rightarrow x a, x \rightarrow x^{-1}$ of $G$ onto itself $(a \in G$ ). Note that $|T|=|G|$. A set $X \subset G$ is said to be almost invariant if $|\tau X \triangle X|<|G|$ for each $\tau \in T$. The family of almost invariant subsets of $G$ forms an algebra.

LEMMA 1. There exists a family of subsets $\left\{X_{\nu}\right\}_{\nu \in P}$ of subsets of $G$ such that:

(i) $|P|=|G|$;

(ii) the sets $X_{\nu}$ are mutually disjoint;

(iii) $\cup\left\{X_{\nu}: \nu \in P_{0}\right\}$ is almost invariant for each subset $P_{0}$ of $P$;

(iv) $\left|X_{\nu}\right|=|G|$ for each $\nu \in P$.

Proof. Let $\omega$ be the first ordinal number with cardinality $|G|$.

Let $\left\{\tau_{\alpha}\right\}_{1 \leqslant \alpha<\omega}$ be a well ordering of $T$. For $1 \leqslant \alpha<\omega, x \in G$ set $C_{\alpha}(x)$ $=\left\{\tau_{\beta_{1}}^{\epsilon_{1} \circ \ldots \circ} \ldots \tau_{\beta_{n}}^{\epsilon_{n}} x: 1 \leqslant \beta_{k} \leqslant \alpha, \epsilon_{k}=1\right.$ or $-1, k=1,2, \ldots, n$, and $n$ $=1,2, \ldots\}$. Note that $x \in C_{\alpha}(x)$; if $\beta \leqslant \alpha$ then $\tau_{\beta}\left(C_{\alpha}(x)\right)=C_{\alpha}(x)$ and $\left|C_{\alpha}(x)\right|<|G|$. (The last inequality holds since $|G|>\aleph_{0}$.) A transfinite double sequence $\chi_{\beta}^{\alpha}, 1 \leqslant \beta \leqslant \alpha<\omega$, in $G$ can be constructed such that the sets $C_{\alpha}\left(x_{\beta}^{\alpha}\right), \quad 1 \leqslant \beta \leqslant \alpha<\omega$, are mutually disjoint. Let $P=\{\nu: \nu$ ordinal number, $1 \leqslant \nu<\omega\}$ and set $X_{\nu}=\cup\left\{C_{\alpha}\left(x_{\nu}^{\alpha}\right): \nu \leqslant \alpha<\omega\right\}$. Then $\left\{X_{\nu}\right\}_{\nu \in P}$ is what we want. The details can be found on pp. 218-219 of [6].

Lemma 2. There exists a collection of subsets of $P,\left\{P_{\theta}\right\}_{\theta \in \Theta}$ such that:

(i) $|\Theta|=2^{|G|}$,

(ii) $\cap \cap_{k=1}^{n} P_{\theta_{k}}^{\epsilon_{k}} \neq \varnothing$ for each finite sequence $\theta_{1}, \ldots, \theta_{n}$ of distinct elements of $\Theta$ and for $\epsilon_{k}=1$ or ${ }^{\prime}$. (If $A \subset P, A^{1}=A, A^{\prime}=P \backslash A$.)

In $[6$, p. 220] the above lemma is proved for the case that $|P|=|G|=c$. Their proof also works here.

Proof of Theorem 2. For each $\theta \in \Theta$, set $E_{\theta}=\cup\left\{X_{\nu}: \nu \in P_{\theta}\right\}$. Then, by Lemmas 1 and 2, for each sequence $\theta_{1}, \ldots, \theta_{n}$ of distinct elements in $\Theta$ and for $\epsilon_{k}=1$ or ',$\cap_{k=1}^{n} E_{\theta_{k}}^{\epsilon_{k}}$ is almost invariant and with cardinality equal to $|G|$. (Here $E_{\theta_{k}}{ }^{\prime}=G \backslash E_{\theta_{k}}$.) Note that for each finite sequence of distinct elements in $\Theta$ the collection $\left\{\cap_{k=1}^{n} E_{\theta_{k}}^{\epsilon_{k}}: \epsilon_{k}=1\right.$ or ' $\}$ forms a partition of $G$ 
into $2^{n}$ disjoint sets. Let $\mathcal{E}$ be the family of sets consisting of the empty set and sets of the form

$$
E=\bigcup\left\{\bigcap_{k=1}^{n} E_{\theta_{k}}^{\epsilon_{k}}:\left(\epsilon_{1}, \ldots, \epsilon_{n}\right) \in \Gamma\right\}
$$

where $\Gamma$ is a subset of $\{1,\}^{n}, \theta_{1}, \ldots, \theta_{n}$ is a sequence of distinct elements in $\Theta$ and $n=1,2, \ldots$ Suppose $E$ is as in (1) and $\theta_{1}, \ldots, \theta_{n}, \theta_{n+1}, \ldots, \theta_{m}$ is a sequence of distinct elements from $\Theta$. Then $E$ can also be written as

$$
E=\bigcup\left\{\bigcap_{k=1}^{m} E_{\theta_{k}}^{\epsilon_{k}}:\left(\epsilon_{1}, \ldots, \epsilon_{n}, \ldots, \epsilon_{m}\right) \in \Gamma^{-}\right\}
$$

where $\Gamma^{-} \subset\left\{1,{ }^{\prime}\right\}^{m}$ is defined by $\left(\epsilon_{1}, \ldots, \epsilon_{m}\right) \in \Gamma^{-}$if and only if $\left(\epsilon_{1}, \ldots, \epsilon_{n}\right) \in \Gamma$. Using this remark it is easy to see that $\varepsilon$ is an algebra (of almost invariant subsets of $G$ ).

Denote the set of all functions from $\Theta$ to the closed interval $[0,1]$ by $Z$. Note that $|Z|=2^{2^{|G|}}$. For each $\xi \in Z$ set $\phi_{\xi}\left(E_{\theta}\right)=\xi(\theta)$ and $\phi_{\xi}\left(E_{\theta}^{\prime}\right)=1-$ $\xi(\theta)$. Extend $\phi_{\xi}$ to $\mathcal{E}$ as follows: $\phi_{\xi}(\varnothing)=0, \phi_{\xi}(E)=\sum\left\{\Pi_{k=1}^{n} \phi_{\xi}\left(E_{\theta_{k}}^{\epsilon_{k}}\right)\right.$ : $\left.\left(\epsilon_{1}, \ldots, \epsilon_{n}\right) \in \Gamma\right\}$ if $E$ is as in (1). By (2) it is easy to see that $\phi_{\xi}$ is a well-defined finitely additive measure on $\varepsilon$ and $\phi_{\xi}(G)=1$.

Let $\mathscr{F}=\{F \subset G:|F \triangle E|<|G|$ for some $E \in \mathcal{E}\}$. Using the fact that each $E \in \mathcal{E}$ is almost invariant one sees:

(3) If $E \in \mathcal{E}$ and $|F \triangle E|<|G|$ then $|\tau F \triangle E|<|G|$ for each $\tau \in T$; therefore $\mathscr{F}$ is closed under $T$.

Extend $\phi_{\xi}$ to $\mathscr{F}$ by setting $\phi_{\xi}(F)=\phi_{\xi}(E)$ if $|F \triangle E|<|G|, E \in \mathcal{E} . \phi_{\xi}$ is well defined on $\mathscr{F}$ since if $\left|F \triangle E_{1}\right|<|G|,\left|F \triangle E_{2}\right|<|G|, \quad E_{i} \in \mathcal{E}$, then $\left|E_{1} \triangle E_{2}\right|<|G|$ and hence $E_{1}=E_{2}$ by (2), Lemma 2(ii) and the definition of $E_{\theta} . \phi_{\xi}$ is clearly finitely additive on $\mathscr{F}$ and, by (3), if $F \in \mathcal{F}$ and $\tau \in T$, $\phi_{\xi}(\tau F)=\phi_{\xi}(F)$.

Let $J=\left\{\sum_{i=1}^{k} c_{i} \chi_{F_{i}}: F_{i} \in \mathscr{F}, c_{i} \in R\right.$ and $\left.k=1,2, \ldots\right\}$ be the vector subspace of $m(G)$ spanned by the characteristic functions $\chi_{F}, F \in \mathcal{F}$. If $f \in J$, $x \in G$ then $l_{x} f, r_{x} f, f^{\sim}$ belong to $J$. Extend $\phi_{\xi}$ to $J$ in the natural way: $\phi_{\xi}\left(\sum_{i=1}^{k} c_{i} \chi_{F_{i}}\right)=\sum_{i=1}^{k} c_{i} \phi_{\xi}\left(F_{i}\right)$. Then $\phi_{\xi}$ is a two-sided invariant mean on $J$ which is also inversion invariant.

Now set $K=\left\{\lambda: \lambda \in m(G)^{*},\|\lambda\|=1, \lambda \geqslant 0, \lambda \mid J=\phi_{\xi}\right\}$. Then $K$ is a nonempty $\omega^{*}$-compact convex subset of $m(G)^{*}$ and $l_{x}^{*} K \subset K, r_{x}^{*} K \subset K$ for each $x \in G$. By Day's fixed point theorem [4] there exist $\lambda_{\xi}^{\prime}, \lambda_{\xi}^{\prime \prime} \in K$ such that $l_{x}^{*} \lambda_{\xi}^{\prime}=\lambda_{\xi}^{\prime}$ and $r_{x}^{*} \lambda_{\xi}^{\prime \prime}=\lambda_{\xi}^{\prime \prime}$, i.e., $\lambda_{\xi}^{\prime} \in M L(G), \lambda_{\xi}^{\prime \prime} \in M R(G)$ and $\lambda_{\xi}^{\prime} \mid J=\phi_{\xi}$, $\lambda_{\xi}^{\prime \prime} \mid J=\phi_{\xi}$. (Note that this is the only place the fact that $G$ is amenable is used.) Define $\lambda_{\xi}$ as follows: $\lambda_{\xi}(f)=\lambda_{\xi}^{\prime}\left(f^{\prime}\right)$ where $f^{\prime}(x)=\lambda_{\xi}^{\prime \prime}\left(l_{x} f\right)(x \in G)$. Then $\lambda_{\xi} \in M(G)$ and $\lambda_{\xi} \mid J=\phi_{\xi}$. Finally set $\mu_{\xi}(f)=\frac{1}{2} \lambda_{\xi}(f+f), f \in m(G)$. Then $\mu_{\xi} \in M^{\sim}(G), \mu_{\xi} \mid J=\phi_{\xi}$ and, hence, the proof of Theorem 2 is completed.

REMARKS. 1. The following shorter and more direct proof of Theorem 1 is provided by the referee. Let $G$ be as in Theorem 2. Construct $\left\{X_{\nu}\right\}_{\nu \in P}$ as in Lemma 1 . Let $\eta$ be a finitely additive probability measure on the power sets of $P$. (Since $\eta$ can be considered as a probability measure on $\beta P$, the 
Stone-Čech compactification of the discrete set $P$, one sees that there are $2^{2^{|G|}}$ such $\eta$ 's.) Let $\mathscr{F}$ be the family of all sets of the form $E_{Q}=\cup\left\{X_{\nu}: \nu \in Q\right\}$, $Q \subset P$. Without loss of generality one may assume that $\cup\left\{X_{\nu}: \nu \in P\right\}=G$ and, hence, $\mathscr{F}$ is a $\sigma$-algebra of almost invariant subsets of $G$. Now set $\phi\left(E_{Q}\right)=\eta(Q)$. Extend $\phi$ to an element in $M^{\sim}(G)$ as in the proof of Theorem $2^{2}$

2. In a countably infinite group a set $A$ is almost invariant if and only if $\tau A$ $\triangle A$ is finite for each $\tau \in T$. In the additive group of integers $\mathbf{Z}$ an almost invariant set is either finite or is a complement of a finite set. Therefore Lemma 1 does not hold for $\mathbf{Z}$. On the other hand, it is not hard to see that if $G$ is a countably infinite locally finite group then Lemma 1 holds, e.g., [8, p. 19].

3. For a group $G$, each $\tau \in T$ can be extended to a homeomorphism of $\beta G$ onto $\beta G$ which again will be denoted by $\tau$. A set $K \subset \beta G$ is said to be invariant if $\tau K \subset K$ for each $\tau \in T$. Joe Rosenblatt has recently communicated to us the following fact: If $G$ is an uncountable group, then using the existence of $\left\{E_{\theta}\right\}_{\theta \in \Theta}$ it can be shown that $\beta G$ contains $2^{2^{|G|}}$ mutually disjoint closed invariant sets. If, in addition, $G$ is amenable, then there exist $2^{2^{|G|}}$ elements in $M^{\sim}(G)$ with mutually disjoint supports. The latter fact also follows from Theorem 2. Indeed if $\xi, \zeta: \Theta \rightarrow\{0,1\}, \xi \neq \eta$, then there exists $E_{\theta}$ such that $\mu_{\xi}\left(E_{\theta}\right) \neq \mu_{\zeta}\left(E_{\theta}\right)$ and hence support $\mu_{\xi} \cap$ support $\mu_{\eta}=\varnothing$. We are unable to decide whether there are $2^{c}$ mutually singular elements in $M^{\sim}(G)$ if $G$ is countably infinite. In [1] we are able to show that $M L(G)$ contains $2^{c}$ mutually singular elements.

\section{REFERENCES}

1. C. Chou, On the size of the set of left invariant means on a semigroup, Proc. Amer. Math. Soc. 23 (1969), 199-205. MR 40 \#10.

2. On topologically invariant means on a locally compact group, Trans. Amer. Math. Soc. 151 (1970), 443-456. MR 42 \#675.

3. M. M. Day, Amenable semigroups, Illinois J. Math. 1 (1957), 509-544. MR 19, 1067.

4. _ Fixed-point theorems for compact convex sets, Illinois J. Math. 5(1961), 585-590. MR 25 \# 1547.

5. E. Granirer, On amenable semigroups with a finite-dimensional set of invariant means, I, II, Illinois J. Math. 7 (1963), 32-58. MR 26 \#1744; \#1745.

6. E. Hewitt and K. A. Ross, Abstract harmonic analysis. Vol. 1: Structure of topological groups. Integration theory, group representations, Die Grundlehren der math. Wissenschaften, Band 115, Academic Press, New York; Springer-Verlag, Berlin, 1963. MR 28 \# 158.

7. S. Kakutani and J. C. Oxtoby, Construction of a non-separable invariant extension of the Lebesgue measure space, Ann. of Math. (2)52 (1950), 580-590. MR 12, 246.

8. D. E. Cohen, Groups of cohomological dimension one, Lecture Notes in Math., vol. 245, Springer-Verlag, Berlin, 1972.

Department of Mathematics, State University of New York at Buffalo, Amherst, New YORK 14226

\footnotetext{
${ }^{2}$ We wish to thank the referee for this and other useful comments.
} 